Review Article

\title{
Solving Higher-Order Fractional Differential Equations by the Fuzzy Generalized Conformable Derivatives
}

\author{
Atimad Harir (D), Said Melliani, and Lalla Saadia Chadli \\ Laboratory of Applied Mathematics and Scientific Computing, Sultan Moulay Slimane University, P.O. Box 523, \\ Beni Mellal 23000, Morocco \\ Correspondence should be addressed to Atimad Harir; atimad.harir@gmail.com
}

Received 11 February 2021; Accepted 13 May 2021; Published 27 May 2021

Academic Editor: Francesco Carlo Morabito

Copyright ( 2021 Atimad Harir et al. This is an open access article distributed under the Creative Commons Attribution License, which permits unrestricted use, distribution, and reproduction in any medium, provided the original work is properly cited.

In this paper, the generalized concept of conformable fractional derivatives of order $q \in(n, n+1]$ for fuzzy functions is introduced. We presented the definition and proved properties and theorems of these derivatives. The fuzzy conformable fractional differential equations and the properties of the fuzzy solution are investigated, developed, and proved. Some examples are provided for both the new solutions.

\section{Introduction}

A closed-form solution for nonlinear fractional differential equations (FDEs) plays a significant role in understanding the qualitative as well as quantitative features of complex physical phenomena. The nonlinear FDEs appear in different sciences and engineering problems such as control theory, signal processing, finance, electricity, mechanics, plasma physics, stochastic dynamical system, economics, and electrochemistry [1-7]. A fuzzy fractional differentiation and fuzzy integration operators have different kinds of definitions that we can mention, the fuzzy Riemann-Liouville definition $[8,9]$, the fuzzy Caputo definition $[9,10]$, and so on. Lately, Khalid et al. [11] introduced a new simple definition of the fractional derivative named the conformable fractional derivative, which can redress shortcomings of the other definitions, and this new definition satisfies formulas of derivative of product and quotient of two functions $[12,13]$. Harir et al. [14] introduced the fuzzy generalized conformable fractional derivative, which generalized and extended the concept of Hukuhara differentiability for set-valued mappings to the class of fuzzy mapping $[15,16]$.

Our objective of this article is to present a generalized concept of conformable fractional derivative of order $q \in(n, n+1], n \in \mathbb{N}$ for fuzzy functions. Then, we have investigated in more detail some new properties of these derivatives and we have proved some useful related theorems. We interpret fuzzy conformable fractional differential equations using this concept. We introduce new definitions of solutions. Two examples are provided.

\section{Preliminaries}

Let us denote by $\mathbb{R}_{\mathscr{F}}=\{u: \mathbb{R} \longrightarrow[0,1]\}$ the class of fuzzy subsets of the real axis satisfying the following properties $[13,17]$ :

(i) $u$ is normal, i.e., there exists an $x_{0} \in \mathbb{R}$ such that $u\left(x_{0}\right)=1$.

(ii) $u$ is fuzzy convex, i.e., for $x, y \in \mathbb{R}$ and $0<\lambda \leq 1$,

$$
u(\lambda x+(1-\lambda) y) \geq \min [u(x), u(y)] .
$$

(iii) $u$ is upper semicontinuous.

(iv) $[u]^{0}=c\{x \in \mathbb{R} \mid u(x)>0\}$ is compact.

Then, $\mathbb{R}_{\mathscr{F}}$ is called the space of fuzzy numbers. Obviously, $\quad \mathbb{R} \subset \mathbb{R}_{\mathscr{F}}$. For $\quad 0<\alpha \leq 1 \quad$ denoting $[u]^{\alpha}=\{x \in \mathbb{R} \mid u(x) \geq \alpha\}$, then from (i) to (iv), it follows that the $\alpha$-level set $[u]^{\alpha} \in P_{K}(\mathbb{R})$ for all $0 \leq \alpha \leq 1$ is a closed bounded interval which is denoted by $[u]^{\alpha}=\left[u_{1}^{\alpha}, u_{2}^{\alpha}\right]$. By $P_{K}(\mathbb{R})$, we denote the family of all nonempty compact 
convex subsets of $\mathbb{R}$ and define the addition and scalar multiplication in $P_{K}(\mathbb{R})$ as usual.

Theorem 1 (see [10]). If $u \in \mathbb{R}_{\mathscr{F}}$, then

(i) $[u]^{\alpha} \in P_{K}(\mathbb{R})$ for all $0 \leq \alpha \leq 1$.

(ii) $[u]^{\alpha_{2}} \subset[u]^{\alpha_{1}}$ for all $0 \leq \alpha_{1} \leq \alpha_{2} \leq 1$.

(iii) $\left\{\alpha_{k}\right\} \subset[0,1]$ is a nondecreasing sequence which converges to $\alpha$; then,

$$
[u]^{\alpha}=\bigcap_{k \geq 1}[u]^{\alpha_{k}} .
$$

Conversely, if $A_{\alpha}=\left\{\left[u_{1}^{\alpha}, u_{2}^{\alpha}\right] ; \alpha \in(0,1]\right\}$ is a family of closed real intervals verifying (i) and (ii), then $\left\{A_{\alpha}\right\}$ is defined as a fuzzy number $u \in \mathbb{R}_{\mathscr{F}}$ such that $[u]^{\alpha}=A_{\alpha}$ for $0<\alpha \leq 1$ and $[u]^{0}=\overline{\mathrm{U}_{0<\alpha \leq 1} A_{\alpha}} \subset A_{0}$.

Lemma 1 (see [18]). Let $u, v: \mathbb{R} \longrightarrow[0,1]$ be the fuzzy sets. Then, $u=v$ if and only if $[u]^{\alpha}=[v]^{\alpha}$ for all $\alpha \in[0,1]$.

The following arithmetic operations on fuzzy numbers are well known and frequently used below [17]. If $u, v \in \mathbb{R}_{\mathscr{F}}$, then

$$
\begin{aligned}
{[u+v]^{\alpha} } & =\left[u_{1}^{\alpha}+v_{1}^{\alpha}, u_{2}^{\alpha}+v_{2}^{\alpha}\right], \\
& {[\lambda u]^{\alpha}=\lambda[u]^{\alpha}= \begin{cases}{\left[\lambda u_{1}^{\alpha}, \lambda u_{2}^{\alpha}\right],} & \text { if } \lambda \geq 0, \\
{\left[\lambda u_{2}^{\alpha}, \lambda u_{1}^{\alpha}\right],} & \text { if } \lambda<0 .\end{cases} }
\end{aligned}
$$

Definition 1. Let $u, v \in \mathbb{R}_{\mathscr{F}}$. If there exists $w \in \mathbb{R}_{\mathscr{F}}$ such as $u=v+w$, then $w$ is called the $H$-difference of $u, v$ and it is denoted as $u \ominus v$.

Theorem 2 (see [19])

(i) Let we denote

$$
\overline{0}= \begin{cases}1, & t=0 \\ 0, & t \neq 0\end{cases}
$$

then $\overline{0} \in \mathbb{R}_{\mathscr{F}}$ is a neutral element with respect to + , i.e., $u+\overline{0}=\overline{0}+u \quad u \in \mathbb{R}_{\mathscr{F}}$.

(ii) With respect to $\overline{0}$, none of $u \in \mathbb{R}_{\mathscr{F}} \backslash \mathbb{R}$ has opposite in $\mathbb{R}_{\mathscr{F}}$.

(iii) For any $a, b \in \mathbb{R}$ with $a, b \geq 0$ or $a, b \leq 0$ and any $u \in \mathbb{R}_{\mathscr{F}}$, we have $(a+b) \cdot u=a \cdot u+b \cdot u$; for general $a, b \in \mathbb{R}$, the above property does not hold.

(iv) For any $\lambda \in \mathbb{R}$ and any $u, v \in \mathbb{R}_{\mathscr{F}}$, we have $\lambda \cdot(u+v)=\lambda \cdot u+\lambda \cdot v$.

(v) For any $\lambda, v \in \mathbb{R}$ and any $u \in \mathbb{R}_{\mathscr{F}}$, we have $\lambda \cdot(\nu \cdot u)=(\lambda \cdot v) \cdot u$.

Define $d: \mathbb{R}_{\mathscr{F}} \times \mathbb{R}_{\mathscr{F}} \longrightarrow \mathbb{R}_{+} \cup\{0\}$ by the following equation:

$$
d(u, v)=\sup _{\alpha \in[0,1]} d_{H}\left([u]^{\alpha},[v]^{\alpha}\right), \quad \text { for all } u, v \in \mathbb{R}_{\mathscr{F}},
$$

where $d_{H}$ is the Hausdorff metric:

$$
d_{H}\left([u]^{\alpha},[v]^{\alpha}\right)=\max \left\{\left|u_{1}^{\alpha}-v_{1}^{\alpha}\right|,\left|u_{2}^{\alpha}-v_{2}^{\alpha}\right|\right\} .
$$

It is well known that $\left(\mathbb{R}_{\mathscr{F}}, d\right)$ is a complete metric space. We list the following properties of $d(u, v)$ [17]:

$$
\begin{aligned}
& d(u+w, v+w)=d(u, v), \\
& d(u, v)=d(v, u), \\
& d(k u, k v)=|k| d(u, v), \\
& d(u, v) \leq d(u, w)+d(w, v),
\end{aligned}
$$

for all $u, v, w \in \mathbb{R}_{\mathscr{F}}$ and $\lambda \in \mathbb{R}$.

Let $\left(A_{k}\right)$ be a sequence in $P_{K}(\mathbb{R})$ converging to $A$. Then, Theorem in [17] gives us an expression for the limit.

Theorem 3 (see [15]). If $d\left(A_{k}, A\right) \longrightarrow 0$ as $k \longrightarrow \infty$, then

$$
A=\bigcap_{\substack{k \geq 1 \\ m \geq k}} \bar{U}_{m}
$$

Let $I=(0, a) \subset \mathbb{R}$ be an interval. We denote by $C\left(I, \mathbb{R}_{\mathscr{F}}\right)$ the space of all continuous fuzzy functions on $I$ which is a complete metric space with respect to the metric

$$
h(u, v)=\sup d(u(t), v(t)) .
$$

\section{Generalized Fuzzy Conformable Fractional Derivatives}

Definition 2 (see [14]). Let $F: I \longrightarrow \mathbb{R}_{\mathscr{F}}$ be a fuzzy function. $q^{\text {th }}$ order "fuzzy conformable fractional derivative" of $F$ is defined by

$$
T_{q}(F)(t)=\lim _{\varepsilon \longrightarrow 0^{+}} \frac{F\left(t+\varepsilon t^{1-q}\right) \ominus F(t)}{\varepsilon}=\lim _{\varepsilon \longrightarrow 0^{+}} \frac{F(t) \ominus F\left(t-\varepsilon t^{1-q}\right)}{\varepsilon},
$$

for all $t>0, q \in(0,1)$. If $F$ is $q$-differentiable in some $I$, and $\lim _{t \rightarrow 0^{+}} T_{q}(F)(t)$ exists, then

$$
T_{q}(F)(0)=\lim _{t \longrightarrow 0^{+}} T_{q}(F)(t)
$$

and the limits exist (in the metric $d$ ).

Remark 1 (see [14]). From the definition, it directly follows that if $F$ is $q$-differentiable, then the multivalued mapping $F_{\alpha}$ is $q$-differentiable for all $\alpha \in[0,1]$ and

$$
T_{q} F_{\alpha}=\left[T_{q}(F)(t)\right]^{\alpha} \text {. }
$$

Here, $T_{q} F_{\alpha}$ is denoted as the conformable fractional derivative of $F_{\alpha}$ of order $q$. The converse result does not hold, since the existence of Hukuhara differences $[x]^{\alpha} \ominus[y]^{\alpha}, \alpha \in[0,1]$, does not imply the existence of $H$-difference $x \ominus y$.

We consider the following definition [14]. 
Definition 3. Let $F: I \longrightarrow \mathbb{R}_{\mathscr{F}}$ be a fuzzy function and $q \in(0,1]$. One says, $F$ is $q_{(1)}$-differentiable at point $t>0$ if there exists an element $T_{q}(F)(t) \in \mathbb{R}_{\mathscr{F}}$ such that for all $\varepsilon>0$ sufficiently near to 0 , there exist $F\left(t+\varepsilon t^{1-q}\right) \ominus F(t), F(t) \ominus F\left(t-\varepsilon t^{1-q}\right)$ and the limits (in the metric $d)$

$$
\lim _{\varepsilon \longrightarrow 0^{+}} \frac{F\left(t+\varepsilon t^{1-q}\right) \ominus F(t)}{\varepsilon}=\lim _{\varepsilon \longrightarrow 0^{+}} \frac{F(t) \ominus F\left(t-\varepsilon t^{1-q}\right)}{\varepsilon}=T_{q}(F)(t) .
$$

$F$ is $q_{(2)}$-differentiable at $t>0$ if for all $\varepsilon<0$ sufficiently near to 0 , there exist $F\left(t+\varepsilon t^{1-q}\right) \ominus F(t), F(t) \ominus F\left(t-\varepsilon t^{1-q}\right)$

$$
\lim _{\varepsilon \longrightarrow 0^{-}} \frac{F\left(t+\varepsilon t^{1-q}\right) \ominus F(t)}{\varepsilon}=\lim _{\varepsilon \longrightarrow 0^{-}} \frac{F(t) \ominus F\left(t-\varepsilon t^{1-q}\right)}{\varepsilon}=T_{q}(F)(t) .
$$

If $F$ is $q_{(n)}$-differentiable at $t>0$, we denote its $q$-derivatives, for $n=1,2$.

Definition 4. Let $F: I \longrightarrow \mathbb{R}_{\mathscr{F}}$ be a fuzzy function and now we introduce definitions and theorems for $q \in(n, n+1]$ for some natural number $n$. For the sake of convenience, we concentrate on $q \in(1,2]$ case be $n$-differentiable at $t$, where $t>0$. Then the fuzzy conformable fractional derivative of $f$ of order $q$ is defined by

$$
\begin{aligned}
T_{q}(F)(t) & =\lim _{\mathcal{\varepsilon} \longrightarrow 0^{+}} \frac{F^{([q]-1)}\left(t+\varepsilon t^{([q]-q)}\right) \ominus F^{([q]-1)}(t)}{\varepsilon} \\
& =\lim _{\varepsilon \longrightarrow 0^{+}} \frac{F^{([q]-1)}(t) \ominus F^{([q]-1)}\left(t-\varepsilon t^{([q]-q)}\right)}{\varepsilon},
\end{aligned}
$$

where $q \in(n, n+1]$ and $[q]$ is the smallest integer greater than or equal to $q$ and the limits exist (in the metric $d$ ).

Theorem 4. Let $F: I \longrightarrow \mathbb{R}_{\mathscr{F}}$ and $q \in(1,2]$ and $n, m=1,2$. If $F$ is $(n, m)$-differentiable and $F$ is $q_{(n, m)}$-differentiable, then

$$
T_{q_{(n, m)}} F(t)=t^{2-q} D_{n, m}^{(2)} F(t) .
$$

Remark 2 (see [20]). $F$ is $(n, m)$-differentiable on $I$, if $D_{n}^{1}$ exists on $I$ and it is $(m)$-differentiable on $I$. The second derivatives of $F$ are denoted by $D_{n, m}^{(2)} F(t)$ for $n, m=1,2$.

Proof. We present the details only for $n=m=1$, since the other case is analogous. Let $h=\varepsilon t^{2-q}$ in Definition 4 , then $\varepsilon=t^{q-2} h$. Therefore, if $\varepsilon>0$ and $\alpha \in[0,1]$, we have

$$
\left[D_{1}^{1} F\left(t+\varepsilon t^{2-q}\right) \ominus D_{1}^{1} F(t)\right]^{\alpha}=\left[\left(f_{1}^{\alpha}\right)^{\prime}\left(t+\varepsilon t^{2-q}\right)-\left(f_{1}^{\alpha}\right)^{\prime}(t),\left(f_{2}^{\alpha}\right)^{\prime}\left(t+\varepsilon t^{2-q}\right)-\left(f_{2}^{\alpha}\right)^{\prime}(t)\right] .
$$

Dividing by $\varepsilon$, we have

$$
\frac{\left[D_{1}^{1} F\left(t+\varepsilon t^{2-q}\right) \ominus D_{1}^{1} F(t)\right]^{\alpha}}{\varepsilon}=\left[\frac{\left(f_{1}^{\alpha}\right)^{\prime}\left(t+\varepsilon t^{2-q}\right)-\left(f_{1}^{\alpha}\right)^{\prime}(t)}{\varepsilon}, \frac{\left(f_{2}^{\alpha}\right)^{\prime}\left(t+\varepsilon t^{2-q}\right)-\left(f_{2}^{\alpha}\right)^{\prime}(t)}{\varepsilon}\right],
$$

and passing to the limit

$$
\begin{aligned}
\lim _{\varepsilon \longrightarrow 0^{+}} \frac{\left[D_{1}^{1} F\left(t+\varepsilon t^{2-q}\right) \ominus D_{1}^{1} F(t)\right]^{\alpha}}{\varepsilon} & =\lim _{\varepsilon \longrightarrow 0^{+}}\left[\frac{\left(f_{1}^{\alpha}\right)^{\prime}\left(t+\varepsilon t^{2-q}\right)-\left(f_{1}^{\alpha}\right)^{\prime}(t)}{\varepsilon}, \frac{\left(f_{2}^{\alpha}\right)^{\prime}\left(t+\varepsilon t^{2-q}\right)-\left(f_{2}^{\alpha}\right)^{\prime}(t)}{\varepsilon}\right] \\
& =\lim _{h \longrightarrow 0^{+}}\left[\frac{\left(f_{1}^{\alpha}\right)^{\prime}(t+h)-\left(f_{1}^{\alpha}\right)^{\prime}(t)}{t^{q-2} h}, \frac{\left(f_{2}^{\alpha}\right)^{\prime}(t+h)-\left(f_{2}^{\alpha}\right)^{\prime}(t)}{t^{q-2} h}\right] \\
& =t^{2-q} \lim _{h \longrightarrow 0^{+}}\left[\frac{\left(f_{1}^{\alpha}\right)^{\prime}(t+h)-\left(f_{1}^{\alpha}\right)^{\prime}(t)}{h}, \frac{\left(f_{2}^{\alpha}\right)^{\prime}(t+h)-\left(f_{2}^{\alpha}\right)^{\prime}(t)}{h}\right] \\
& =t^{2-q}\left[\left(f_{1}^{\alpha}\right)^{\prime \prime}(t),\left(f_{2}^{\alpha}\right)^{\prime \prime}(t)\right] .
\end{aligned}
$$

Similarly, we obtain 


$$
\begin{aligned}
& \frac{\left[D_{1}^{1} F(t) \ominus D_{1}^{1} F\left(t-\varepsilon t^{2-q}\right)\right]^{\alpha}}{\varepsilon}=\left[\frac{\left(f_{1}^{\alpha}\right)^{\prime}(t)-\left(f_{1}^{\alpha}\right)^{\prime}\left(t-\varepsilon t^{2-q}\right)}{\varepsilon}, \frac{\left(f_{2}^{\alpha}\right)^{\prime}(t)-\left(f_{2}^{\alpha}\right)^{\prime}\left(t-\varepsilon t^{2-q}\right)}{\varepsilon}\right], \\
& \text { to the limit and } \varepsilon=t^{q-2} h \text { gives } \quad T_{p_{(n)}}\left(D_{m}^{1} F(t)\right) \neq D_{m}^{1}\left(T_{p_{(n)}} F(t)\right),
\end{aligned}
$$

and passing to the limit and $\varepsilon=t^{q-2} h$ gives $T_{q_{(1,1)}} F(t)=t^{2-q}\left[\left(f_{1}^{\alpha}\right)^{\prime \prime}(t),\left(f_{2}^{\alpha}\right)^{\prime \prime}(t)\right]$.

Theorem 5. Let $0<q, p<1$, and $F$ be a fuzzy function 2 times differentiable on an open real interval I, then the fuzzy conformable derivative obeys to the following

$$
T_{p_{(n)}}\left(T_{q_{(m)}} F(t)\right) \neq T_{q_{(m)}}\left(T_{p_{(n)}} F(t)\right),
$$

where $n, m=1,2$.

Proof. Let $n, m=1,2$. By using Theorem 7 and Theorem 8 in [14], we have the following relation;

$$
\begin{aligned}
T_{p(n)}\left(T_{q_{(m)}} F(t)\right)= & T_{p_{(n)}}\left(t^{1-q} D_{m}^{1} F(t)\right) \\
= & T_{p_{(n)}}\left(t^{1-q}\right) D_{m}^{1} F(t)+t^{1-q} T_{p_{(n)}}\left(D_{m}^{1} F(t)\right) \\
= & t^{1-p}(1-q) t^{-q} D_{m}^{1} F(t) \\
& +t^{1-q} t^{1-p} D_{n}^{1}\left(D_{m}^{1} F(t)\right) \\
= & t^{1-p-q}(1-q) D_{m}^{1} F(t) \\
& +t^{2-q-p}\left(D_{m, n}^{2} F(t)\right) \\
= & t^{1-p-q}\left[(1-q) D_{m}^{1} F(t)+t D_{m, n}^{2} F(t)\right] .
\end{aligned}
$$

On the other hand,

$$
\begin{aligned}
T_{q_{(m)}}\left(T_{p_{(n)}} F(t)\right)= & T_{q_{(m)}}\left(t^{1-p} D_{n}^{1} F(t)\right) \\
= & T_{q_{(m)}}\left(t^{1-p}\right) D_{n}^{1} F(t)+t^{1-} T_{q_{(m)}}\left(D_{n}^{1} F(t)\right) \\
= & t^{1-q}(1-p) t^{-p} D_{n}^{1} F(t) \\
& +t^{1-p} t^{1-q} D_{m}^{1}\left(D_{n}^{1} F(t)\right) \\
= & t^{1-p-q}(1-p) D_{n}^{1} F(t) \\
& +t^{2-q-p}\left(D_{n, m}^{2} F(t)\right) \\
= & t^{1-p-q}\left[(1-p) D_{n}^{1} F(t)+t D_{n, m}^{2} F(t)\right] .
\end{aligned}
$$

It follows that

$$
\begin{aligned}
& t^{1-p-q}\left[(1-q) D_{m}^{1} F(t)+t D_{m, n}^{2} F(t)\right] \\
& \neq t^{1-p-q}\left[(1-p) D_{n}^{1} F(t)+t D_{n, m}^{2} F(t)\right],
\end{aligned}
$$

for all $n, m=1,2$. The proof is complete.

Lemma 2. Let $0<q<1$ and $F$ be a fuzzy function 2 times differentiable on an open real interval $I$, then the fuzzy conformable derivative obeys the following where $n, m=1,2$.

Proof. Let $n, m=1,2$. By using Theorems 7 and 8 in [14], we have the following relation:

$$
\begin{aligned}
T_{p_{(n)}}\left(D_{m}^{1} F(t)\right) & =T_{p_{(n)}}\left(D_{m}^{1} F(t)\right) \\
& =t^{1-p} D_{n}^{1}\left(D_{m}^{1} F(t)\right) \\
& =t^{1-p}\left(D_{m, n}^{2} F(t)\right) .
\end{aligned}
$$

On the other hand,

$$
\begin{aligned}
D_{m}^{1}\left(T_{p_{(n)}} F(t)\right) & =D_{m}^{1}\left(t^{1-p} D_{n}^{1} F(t)\right) \\
& =D_{m}^{1}\left(t^{1-p}\right) D_{n}^{1} F(t)+t^{1-p} D_{m}^{1}\left(D_{n}^{1} F(t)\right) \\
& =(1-p) t^{-p} D_{n}^{1} F(t)+t^{1-p} D_{m}^{1}\left(D_{n}^{1} F(t)\right) \\
& =t^{-p}(1-p) D_{n}^{1} F(t)+t^{1-p}\left(D_{n, m}^{2} F(t)\right) .
\end{aligned}
$$

It follows that

$$
t^{-p}(1-p) D_{n}^{1} F(t)+t^{1-p}\left(D_{n, m}^{2} F(t)\right) \neq t^{1-p}\left(D_{m, n}^{2} F(t)\right),
$$

for all $n, m=1,2$. The proof is complete.

Theorem 6. Let $F: I \longrightarrow \mathbb{R}_{\mathscr{F}}, 1<q \leq 2$ and $p=q-1$. Then the fuzzy conformable fractional derivative of order $q$, where $D_{n}^{1} F(t)$ exists, is defined by

$$
\left(T_{q_{(n, m)}} F\right)(t)=\left(T_{p_{(m)}} D_{n}^{1} F\right)(t)
$$

where $n, m=1,2$.

Proof. We present the details only for the case $n=m=1$, since the other case is analogous. By using Theorem 8 in [14] and Theorem 2.2 in [20], we have

$$
\begin{aligned}
\left(T_{p_{(1)}} D_{1}^{1} F\right)(t) & =t^{1-p}\left(D_{1}^{1}\left(D_{1}^{1} F\right)\right)(t) \\
& =t^{1-p}\left(D_{(1,1)}^{2} F\right)(t) \\
& =t^{1-(q-1)}\left(D_{(1,1)}^{2} F\right)(t) \\
& =t^{2-q}\left(D_{(1,1)}^{2} F\right)(t),
\end{aligned}
$$

and of Theorem 4, it is that

$$
T_{q_{(1,1)}} F(t)=t^{2-q} D_{1,1}^{(2)} F(t) .
$$

Remark 3. $F$ is $q_{(n, m)}$-differentiable on $I$, if $D_{n}^{1}$ exists on $I$ and it is $p_{(m)}$-differentiable on $I$ and $p_{(m)}=q_{(n, m)}-1$. The 
q-differentiable (conformable fractional derivatives of order $q \in(0,1])$ of $F$ is denoted by $T_{q_{(n, m)}} F(t)$ for $n, m=1,2$.

Theorem 7. Let $1<q \leq 2$ and $p=q-1$. Then the fuzzy conformable fractional derivative of $F$ order $q$, where $D_{1}^{1} F: I \longrightarrow \mathbb{R}_{\mathscr{F}} \quad$ or $\quad D_{2}^{1} F: I \longrightarrow \mathbb{R}_{\mathscr{F}}$ exists, where $[F(t)]^{\alpha}=\left[f_{1}^{\alpha}(t), f_{2}^{\alpha}(t)\right]$, is defined by

(i) If $D_{1}^{1} F$ is $p_{(1)}$-differentiable, then $\left(f_{1}^{\alpha}\right)^{\prime}(t)$ and $\left(f_{2}^{\alpha}\right)^{\prime}(t)$ are $p$-differentiable and

$$
\begin{aligned}
{\left[T_{q_{(1,1)}} F(t)\right]^{\alpha} } & =\left[T_{p_{(1)}} D_{1}^{1} F(t)\right]^{\alpha} \\
& =\left[T_{p}\left(f_{1}^{\alpha}\right)^{\prime}(t), T_{p}\left(f_{2}^{\alpha}\right)^{\prime}(t)\right] .
\end{aligned}
$$

(ii) If $D_{1}^{1} F$ is $p_{(2)}$-differentiable, then $\left(f_{1}^{\alpha}\right)^{\prime}(t)$ and $\left(f_{2}^{\alpha}\right)^{\prime}(t)$ are $p$-differentiable and

$$
\begin{aligned}
{\left[T_{q_{(1,2)}} F(t)\right]^{\alpha} } & =\left[T_{p_{(2)}} D_{1}^{1} F(t)\right]^{\alpha} \\
& =\left[T_{p}\left(f_{2}^{\alpha}\right)^{\prime}(t), T_{p}\left(f_{1}^{\alpha}\right)^{\prime}(t)\right] .
\end{aligned}
$$

(iii) If $D_{2}^{1} F$ is $p_{(1)}$-differentiable, then $\left(f_{1}^{\alpha}\right)^{\prime}(t)$ and $\left(f_{2}^{\alpha}\right)^{\prime}(t)$ are $p$-differentiable and

$$
\begin{aligned}
{\left[T_{q_{(2,1)}} F(t)\right]^{\alpha} } & =\left[T_{p_{(1)}} D_{2}^{1} F(t)\right]^{\alpha} \\
& =\left[T_{p}\left(f_{2}^{\alpha}\right)^{\prime}(t), T_{p}\left(f_{1}^{\alpha}\right)^{\prime}(t)\right] .
\end{aligned}
$$

(iv) If $D_{2}^{1} F$ is $p_{(2)}$-differentiable, then $\left(f_{1}^{\alpha}\right)^{\prime}(t)$ and $\left(f_{2}^{\alpha}\right)^{\prime}(t)$ are $p$-differentiable and

$$
\begin{aligned}
{\left[T_{q_{(2,2)}} F(t)\right]^{\alpha} } & =\left[T_{p_{(2)}} D_{2}^{1} F(t)\right]^{\alpha} \\
& =\left[T_{p}\left(f_{1}^{\alpha}\right)^{\prime}(t), T_{p}\left(f_{2}^{\alpha}\right)^{\prime}(t)\right] .
\end{aligned}
$$

Proof. We present the details only for $n=m=1$, since the other cases are analogous. If $\varepsilon>0$ and $\alpha \in[0,1]$, we have

$$
\begin{aligned}
& {\left[D_{1}^{1} F\left(t+\varepsilon t^{1-p}\right) \ominus D_{1}^{1} F(t)\right]^{\alpha}} \\
& =\left[\left(f_{1}^{\alpha}\right)^{\prime}(t)\left(t+\varepsilon t^{1-p}\right)-\left(f_{1}^{\alpha}\right)^{\prime}(t)(t),\left(f_{2}^{\alpha}\right)^{\prime}(t)\left(t+\varepsilon t^{1-p}\right)\right. \\
& \left.\quad-\left(f_{2}^{\alpha}\right)^{\prime}(t)(t)\right] .
\end{aligned}
$$

Dividing by $\varepsilon$, we have

$$
\begin{aligned}
{\left[D_{1}^{1} F\left(t+\varepsilon t^{1-p}\right) \ominus D_{1}^{1} F(t)\right]^{\alpha} } \\
=\left[\left(f_{1}^{\alpha}\right)^{\prime}(t)\left(t+\varepsilon t^{1-p}\right)-\left(f_{1}^{\alpha}\right)^{\prime}(t)(t),\left(f_{2}^{\alpha}\right)^{\prime}(t)\left(t+\varepsilon t^{1-p}\right)\right. \\
\left.\quad-\left(f_{2}^{\alpha}\right)^{\prime}(t)(t)\right] .
\end{aligned}
$$

Dividing by $\varepsilon$, we have

$$
\frac{\left[D_{1}^{1} F\left(t+\varepsilon t^{1-p}\right) \ominus D_{1}^{1} F(t)\right]^{\alpha}}{\varepsilon}=\left[\frac{\left(f_{1}^{\alpha}\right)^{\prime}\left(t+\varepsilon t^{1-p}\right)-\left(f_{1}^{\alpha}\right)^{\prime}(t)}{\varepsilon}, \frac{\left(f_{2}^{\alpha}\right)^{\prime}\left(t+\varepsilon t^{1-p}\right)-\left(f_{2}^{\alpha}\right)^{\prime}(t)}{\varepsilon}\right] .
$$

Similarly, we obtain

$$
\frac{\left[D_{1}^{1} F(t) \ominus D_{1}^{1} F\left(t-\varepsilon t^{1-p}\right)\right]^{\alpha}}{\varepsilon}=\left[\frac{\left(f_{1}^{\alpha}\right)^{\prime}(t)-\left(f_{1}^{\alpha}\right)^{\prime}\left(t-\varepsilon t^{1-p}\right)}{\varepsilon}, \frac{\left(f_{2}^{\alpha}\right)^{\prime}(t)-\left(f_{2}^{\alpha}\right)^{\prime}\left(t-\varepsilon t^{1-p}\right)}{\varepsilon}\right]
$$

and passing to the limit, we have

$$
\left[T_{p_{(1)}} D_{1}^{1} F(t)\right]^{\alpha}=\left[T_{p}\left(f_{1}^{\alpha}\right)^{\prime}(t), T_{p}\left(f_{2}^{\alpha}\right)^{\prime}(t)\right]
$$

and using Theorem 6 gives the theorem.

\section{Fuzzy Conformable Fractional Differential Equations of Order $q$}

In this section, we study the fuzzy conformable fractional differential equations of order $q \in(1,2], p \in(0,1]$ :

$$
\left\{\begin{array}{l}
\frac{\mathrm{d}^{q}}{\mathrm{~d} t^{q}} y(t)+a \frac{\mathrm{d}^{p}}{\mathrm{~d} t^{p}} y(t)+b y(t)=g(t), \quad t \geq 0, \\
y(0)=\sigma_{0}, \\
y^{\prime}(0)=\sigma_{1},
\end{array}\right.
$$

where $a, b>0, \sigma_{0}, \sigma_{1} \in \mathbb{R}_{\mathscr{F}}$, and $g(t)$ is a continuous fuzzy function on some interval $I$. We give the following definition for the solutions of (41). 
Definition 5. Let $y: I \longrightarrow \mathbb{R}_{\mathscr{F}}, 1<q \leq 2,0<p \leq 1$ and $n, m \in\{1,2\} . y$ is a solution $(n, m)$, for problem (41) on $I$, if $D_{n}^{1} y, T_{p_{(n)}} y, T_{q_{(n, m)}} y \quad$ (or $D_{n}^{1} y, T_{p_{(m)}} y$ and $\left.T_{p_{(m)}} D_{n}^{f_{(n)}} y p_{(m)} \stackrel{q_{(n, m)}}{=} q_{(n, m)}-1\right)$ exist on $I$ and

$$
\left\{\begin{array}{l}
T_{q_{(n, m)}} y(t)+a T_{p_{(n)}} y(t)+b y(t)=g(t), \quad t \geq 0, \\
y(0)=\sigma_{0} \\
D_{n}^{1} y(0)=\sigma_{1}
\end{array}\right.
$$

Remark 4. From Theorem 4 and Theorem 8 in [14], it directly follows that $y$ is a solution $(n, m)$, for problem $(41)$ on $I$, if $D_{n}^{1} y$ and $D_{(n, m)}^{2} y$ exist on $I$, and

$$
\left\{\begin{array}{l}
t^{2-q} D_{(n, m)}^{2} y(t)+a t^{1-p} D_{(n)} y(t)+b y(t)=g(t), \quad t \geq 0, \\
y(0)=\sigma_{0}, \\
D_{n}^{1} y(0)=\sigma_{1},
\end{array}\right.
$$

where $q \in(1,2], p \in(0,1]$ and $n, m \in\{1,2\}$.

Therefore, since the fuzzy conformable derivatives of fuzzy processes

$$
\begin{array}{ll}
{\left[T_{p} y(t)\right]^{\alpha}=\left[T_{p} y_{1}^{\alpha}(t), T_{p} y_{2}^{\alpha}(t)\right],} & p \in(0,1], \alpha \in[0,1], \\
{\left[T_{q} y(t)\right]^{\alpha}=\left[T_{q} y_{1}^{\alpha}(t), T_{q} y_{2}^{\alpha}(t)\right],} & q \in(1,2], \alpha \in[0,1],
\end{array}
$$

provided these two intervals define fuzzy numbers $T_{p} y(t), p \in(0,1]$ and $T_{q} y(t), q \in(1,2]$ in $\mathbb{R}_{\mathscr{F}}$, otherwise we apply Definition 2 and Theorem 7 , then we have one of the following cases for $q \in(1,2]$ and $p \in(0,1]$ :

(i) System $(1,1)$

$$
\begin{cases}T_{q} y_{1}^{\alpha}(t)+a T_{p} y_{1}^{\alpha}(t)+b y_{1}^{\alpha}(t)=g_{1}^{\alpha}(t) & y_{1}^{\alpha}(0)=\sigma_{01}^{\alpha},\left(y_{1}^{\alpha}\right)^{\prime}(0)=\sigma_{11}^{\alpha} \\ T_{q} y_{2}^{\alpha}(t)+a T_{p} y_{2}^{\alpha}(t)+b y_{2}^{\alpha}(t)=g_{2}^{\alpha}(t) & y_{2}^{\alpha}(0)=\sigma_{02}^{\alpha},\left(y_{2}^{\alpha}\right)^{\prime}(0)=\sigma_{12}^{\alpha}\end{cases}
$$

(ii) System $(1,2)$

$$
\begin{cases}T_{q} y_{2}^{\alpha}(t)+a T_{p} y_{1}^{\alpha}(t)+b y_{1}^{\alpha}(t)=g_{1}^{\alpha}(t) & y_{1}(0)=\sigma_{01}^{\alpha},\left(y_{1}^{\alpha}\right)^{\prime}(0)=\sigma_{11}^{\alpha} \\ T_{q} y_{1}^{\alpha}(t)+a T_{p} y_{2}^{\alpha}(t)+b y_{2}^{\alpha}(t)=g_{2}^{\alpha}(t) & y_{2}(0)=\sigma_{02}^{\alpha},\left(y_{2}^{\alpha}\right)^{\prime}(0)=\sigma_{12}^{\alpha}\end{cases}
$$

(iii) System $(2,1)$

$$
\begin{cases}T_{q} y_{2}^{\alpha}(t)+a T_{p} y_{2}^{\alpha}(t)+b y_{1}^{\alpha}(t)=g_{1}^{\alpha}(t) & y_{1}(0)=\sigma_{01}^{\alpha},\left(y_{2}^{\alpha}\right)^{\prime}(0)=\sigma_{11}^{\alpha} \\ T_{q} y_{1}^{\alpha}(t)+a T_{p} y_{1}^{\alpha}(t)+b y_{2}^{\alpha}(t)=g_{2}^{\alpha}(t) & y_{2}(0)=\sigma_{02}^{\alpha},\left(y_{1}^{\alpha}\right)^{\prime}(0)=\sigma_{12}^{\alpha}\end{cases}
$$

(iv) System $(2,2)$

$$
\begin{cases}T_{q} y_{1}^{\alpha}(t)+a T_{p} y_{2}^{\alpha}(t)+b y_{1}^{\alpha}(t)=g_{1}^{\alpha}(t) & y_{1}(0)=\sigma_{01}^{\alpha},\left(y_{2}^{\alpha}\right)^{\prime}(0)=\sigma_{11}^{\alpha} \\ T_{q} y_{2}^{\alpha}(t)+a T_{p} y_{1}^{\alpha}(t)+b y_{2}^{\alpha}(t)=g_{2}^{\alpha}(t) & y_{2}(0)=\sigma_{02}^{\alpha},\left(y_{1}^{\alpha}\right)^{\prime}(0)=\sigma_{12}^{\alpha}\end{cases}
$$

where $\left[\sigma_{0}\right]^{\alpha}=\left[\sigma_{01}^{\alpha}, \sigma_{02}^{\alpha}\right],\left[\sigma_{1}\right]^{\alpha}=\left[\sigma_{11}^{\alpha}, \sigma_{12}^{\alpha}\right]$ and $[g(t)]^{\alpha}=\left[g_{1}^{\alpha}(t), g_{2}^{\alpha}(t)\right]$.
Theorem 8. Let $n, m \in\{1,2\}$ and $y=\left[y_{1}, y_{2}\right]$ be a solution $(n, m)$ for problem (41) on I. Then $y_{1}$ and $y_{2}$ solve the associated system $(n, m)$. 
Proof. By using Theorem 4 and Remark 4 and suppose $y$ is the solution $(n, m)$ of problem (41), according to Definition 5 , then $T_{p_{(n)}}, p \in(0,1]$ and $T_{q_{(n, m)}}, q \in(1,2]$ or $\left(t^{1-p} D_{n}^{1}\right.$ and $\left.t^{2-q} D_{n, m}^{2}\right)$ exist and satisfy problem (41). By Theorem 6 in [14], (21) and (25) and substituting $y_{1}, y_{2}$ and their conformable fractional derivatives in problem (41), we get the system $(n, m)$ corresponding to solution $(n, m)$. This completes the proof.

Theorem 9. Let $n, m \in\{1,2\}, p \in(0,1]$ and $y_{1}^{\alpha}$ and $y_{2}^{\alpha}$ solve the system $(n, m)$ on $I$, for all $\alpha \in[0,1]$. Let $[y(t)]^{\alpha}=\left[y_{1}^{\alpha}(t), y_{2}^{\alpha}(t)\right]$. If $y$ has valid cut sets on $I$ and $T_{p_{(m)}} D_{n}^{1}$ exists, then $y$ is a solution $(n, m)$ for fuzzy problem $(41)^{(m)}$.

Proof. Let $q \in(1,2]$ and $[y(t)]^{\alpha}=\left[y_{1}^{\alpha}(t), y_{2}^{\alpha}(t)\right]$ is $q$-differentiable fuzzy function, let $p \in(0,1]$, so by using Theorems 6 and 7 and Theorem 6 in [14] and Remark 4, we can compute $D_{n}^{1} y, T_{p_{(n)}} y$ and $T_{p_{(m)}} D_{n}^{1} y, p_{(m)}=q_{(n, m)}-1$ according to $\left(y_{1}^{\alpha}\right)^{\prime},\left(y_{2}^{\alpha}\right)^{\prime}, t^{2-q}\left(y_{1}^{\alpha}\right)^{p \prime \prime}, t^{2-q}\left(y_{2}^{\alpha}\right)^{\prime \prime}$. Due to the fact that $y_{1}^{\alpha}$ and $y_{2}^{\alpha}$ solve system $(n, m)$, from Definition 5 , then $y$ is a solution $(n, m)$ for equation (41).

\section{Examples}

Example 1. We consider a conformable fractional ordinary differential equation [13]:

$$
\begin{cases}\frac{\mathrm{d}^{3 / 2} y(t)}{\mathrm{d} t^{3 / 2}}=k, & t \geq 0, \\ y(0)=\sigma_{0}, & y^{\prime}(0)=\sigma_{1},\end{cases}
$$

where $k=\sigma_{0}=\sigma_{1}$ are the triangular fuzzy numbers having $\alpha$-cuts $[\alpha-1,1-\alpha]$. If $y$ is solution $(1,1)$ for problem $(49)$ and $p=1 / 2$, then $\left[D_{1}^{1} y(t)\right]^{\alpha}=\left[\left(y_{1}^{\alpha}\right)^{\prime}(t),\left(y_{2}^{\alpha}\right)^{\prime}(t)\right]$ and

$$
\begin{aligned}
{\left[T_{3 / 2_{(1,1)}} y(t)\right]^{\alpha} } & =\left[T_{1 / 2_{(1)}}\left[\left(y_{1}^{\alpha}\right)^{\prime}(t),\left(y_{2}^{\alpha}\right)^{\prime}(t)\right]^{\alpha}\right. \\
& =\left[\frac{\mathrm{d}^{3 / 2}}{\mathrm{~d} t^{3 / 2}} y_{1}^{\alpha}(t), \frac{\mathrm{d}^{3 / 2}}{\mathrm{~d} t^{3 / 2}} y_{2}^{\alpha}(t)\right]^{\alpha},
\end{aligned}
$$

and they satisfy system $(1,1)$ associated with equation (41). Using Theorems 6 and 4, so the conformable fractional system $(1,1)$ has only the following solution:

$$
\begin{aligned}
& y_{1}^{\alpha}(t)=(\alpha-1)\left(\frac{4}{3} t^{3 / 2}+t+1\right) \\
& y_{2}^{\alpha}(t)=(1-\alpha)\left(\frac{4}{3} t^{3 / 2}+t+1\right) .
\end{aligned}
$$

Then, $y(t)=[\alpha-1,1-\alpha]\left((4 / 3) t^{3 / 2}+t+1\right)$ has valid $\alpha$-cuts for $t \geq 0$. By Theorem 2.3 in [20], $y(t)$ is a conformable fractional derivative of order $(3 / 2)_{(1,1)}$ for $t \geq 0$. So $y(t)$ defines a solution $(1,1)$ for $t \geq 0$. For solution $(1,2)$, we deduce

$$
\begin{aligned}
& y_{1}^{\alpha}(t)=(\alpha-1)\left(-\frac{4}{3} t^{3 / 2}+t+1\right), \\
& y_{2}^{\alpha}(t)=(1-\alpha)\left(-\frac{4}{3} t^{3 / 2}+t+1\right),
\end{aligned}
$$

where $y(t)$ has valid $\alpha$-cuts for $t \in[0,0.25]$ and is a conformable fractional derivative of order $(3 / 2)_{(1,2)}$ for $t \in(0,0.25)$. Hence, $y(t)$ gives us a solution on $t \in(0,0.25)$. For solution $(1,2)$, we get

$$
\begin{aligned}
& y_{1}^{\alpha}(t)=(\alpha-1)\left(-\frac{4}{3} t^{3 / 2}-t+1\right), \\
& y_{2}^{\alpha}(t)=(1-\alpha)\left(-\frac{4}{3} t^{3 / 2}-t+1\right),
\end{aligned}
$$

where $y(t)$ has valid $\alpha$-cuts for $t \in[0,0.51]$. We can see $y(t)$ is a solution $(1,2)$ on $(0,0.51)$. Finally, system $(2,2)$ gives

$$
\begin{aligned}
& y_{1}^{\alpha}(t)=(\alpha-1)\left(\frac{4}{3} t^{3 / 2}-t+1\right), \\
& y_{2}^{\alpha}(t)=(1-\alpha)\left(\frac{4}{3} t^{3 / 2}-t+1\right),
\end{aligned}
$$

where $y(t)$ has valid $\alpha$-cuts for all $0 \leq t \leq 0.25$ and defines a solution $(2,2)$ on $t \in(0,0.25)$. Then, we have an example of a fuzzy conformable fractional ordinary differential equation with four solutions.

Example 2. Given a conformable fractional ordinary differential equation $[13,21]$

$$
\left\{\begin{array}{l}
\frac{\mathrm{d}^{3 / 2} y(t)}{\mathrm{d} t^{3 / 2}}=\frac{\mathrm{d}^{1 / 2} y(t)}{\mathrm{d} t^{1 / 2}}, \quad t \geq 0 \\
y(0)=\sigma_{0} \\
y^{\prime}(0)=\sigma_{1}
\end{array}\right.
$$

where $\sigma_{0}$ and $\sigma_{1}$ are the triangular fuzzy numbers having $\alpha$-cuts

$$
\begin{aligned}
& {\left[\sigma_{0}\right]^{\alpha}=[\alpha-1,1-\alpha],} \\
& {\left[\sigma_{1}\right]^{\alpha}=[\alpha, 2-\alpha], \quad \text { for all } \alpha \in[0,1] .}
\end{aligned}
$$

To find solution $(1,1)$, we have

$$
\begin{aligned}
& y_{1}^{\alpha}(t)=-1+\alpha e^{t}, \\
& y_{2}^{\alpha}(t)=-1+(2-\alpha) e^{t},
\end{aligned}
$$

where $y(t)$ has valid $\alpha$-cuts for $t \geq 0$ and $y(t)=-1+\sigma_{1} e^{t}$. From Theorem 2.3 in [20], $y(t)$ is a conformable fractional derivative of order $(3 / 2)_{(1,1)}$ for $t \geq 0$. So $y(t)$ defines a solution $(1,1)$ for $t \geq 0$. For solution $(1,2)$, we deduce

$$
\begin{aligned}
& y_{1}^{\alpha}(t)=-1+e^{t}+(\alpha-1) e^{-t} \\
& y_{2}^{\alpha}(t)=-1+e^{t}+(1-\alpha) e^{-t}
\end{aligned}
$$

We see that $y(t)$ has valid alpha-cut and $y(t)=-1+e^{t}+\sigma_{0} e^{-t}$. From Theorem 2.3 in [20], $y(t)$ is a 
conformable fractional derivative of $\operatorname{order} T_{(3 / 2)_{(2,2)}}$ for $t \geq 0$. Since system $(1,2)$ has only the above solution, then solution $(1,2)$ does not exist. For solution $(2,1)$, we get

$$
\begin{aligned}
& y_{1}^{\alpha}(t)=-1+\alpha e^{t} \\
& y_{2}^{\alpha}(t)=-1+(2-\alpha) e^{t} .
\end{aligned}
$$

$y(t)$ has valid $\alpha$-cuts and $y(t)$ is a conformable fractional derivative of order $(3 / 2)_{(1,1)}$ for $t$. Then, solution $(2,1)$ does not exist. For solution $(2,2)$, we deduce

$$
\begin{aligned}
& y_{1}^{\alpha}(t)=-1+e^{t}+(\alpha-1) e^{-t} \\
& y_{2}^{\alpha}(t)=-1+e^{t}+(1-\alpha) e^{-t}
\end{aligned}
$$

We see that $y(t)$ has valid $\alpha$-cut and $y(t)=-1+e^{t}+\sigma_{0} e^{-t}$. From Theorem 2.3 in [20], $y(t)$ is a conformable fractional derivative of order $(3 / 2)_{(2,2)}$ for all $t \geq 0$. Then $y(t)$ defines a solution for $t \geq 0$. Then, we have a fuzzy conformable fractional ordinary differential equation and two solutions.

\section{Conclusion}

By using the concept of conformable generalized derivative and its extension to fractional derivatives of order $q \in(0,2]$, we show that we have several possibilities or types to define fractional derivatives of order $q \in(0,2]$ of fuzzy-numbervalued functions. Then, we propose a new method to solve fuzzy fractional differential equations based on the selection of conformable derivative types covering all former solutions. With these ideas, the selection of conformable derivative type in each step of deprivation plays a crucial role.

For future research, we will solve the fractional fuzzy conformable partial differential equations [22, 23] by using the proposed method.

\section{Data Availability}

No data were used to support this study.

\section{Conflicts of Interest}

The authors declare that they have no conflicts of interest.

\section{References}

[1] R. Hilfer, Applications of Fractional Calculus in Physics, World Scientific, Singapore, 2000.

[2] A. Kilbas, H. Srivastava, and J. trujillo, Theory and Applications of Fractional Differential Equations. North-holland mathematics studies, Elsevier Science B.V, Amsterdam, The Netherland, 2006.

[3] K. Mayathevar, M. Veluchamy, and B. Subramani, "Fuzzy color histogram equalization with weighted distribution for image enhancement," Optik, vol. 216, Article ID 164927, 2020.

[4] M. Versaci and F. C. Morabito, "Image edge detection: a new approach based on fuzzy entropy and fuzzy divergence," International Journal of Fuzzy Systems, 2021.

[5] M. Versaci, F. C. Morabito, and G. Angiulli, "Adaptive image contrast enhancement by computing distances into a 4- dimensional fuzzy unit hypercube," IEEE Access, vol. 5, pp. 26922-26931, 2017.

[6] B. West, M. Bologna, and P. Grigolini, Physics of Fractal Operators, Springer Science and Business Media, Berlin, Germany, 2012.

[7] L. Zhang, Z. Jia, L. Koefoed, J. Yang, and N. Kasabov, "Remote sensing image enhancement based on the combination of adaptive nonlinear gain and the PLIP model in the NSST domain," Multimedia Tools and Applications, vol. 79, no. 1920, pp. 13647-13665, 2020.

[8] S. Arshad and V. Lupulescu, "On the fractional differential equations with uncertainty," Nonlinear Analysis: Theory, Methods and Applications, vol. 74, no. 11, pp. 3685-3693, 2011.

[9] A. Harir, S. Melliani, and L. S. Chadli, "Fuzzy fractional evolution equations and fuzzy solution operators," Advances in Fuzzy Systems, vol. 2019, Article ID 5734190, 10 pages, 2019.

[10] N. V. Hoa, H. Vu, and T. M. Duc, "Fuzzy fractional differential equations under Caputo-Katugampola fractional derivative approach," Fuzzy Sets and Systems, vol. 375, pp. 70-99, 2019.

[11] R. Khalil, M. Al Horani, A. Yousef, and M. Sababheh, "A new definition of fractional derivative," Journal of Computational and Applied Mathematics, vol. 264, pp. 65-70, 2014.

[12] O. Özkan and A. Kurt, "Exact solutions of fractional partial differential equation systems with conformable derivative," Filomat, vol. 33, no. 5, pp. 1313-1322, 2019.

[13] E. Gökdoğan and A. Gokdogan, "Solution of conformable fractional ordinary differential equations via differential transform method," Optik, vol. 128, pp. 264-273, 2017.

[14] A. Harir, S. Melliani, and L. S. Chadli, "Fuzzy generalized conformable fractional derivative," Advances in Fuzzy Systems, vol. 2020, Article ID 1954975, 7 pages, 2020.

[15] B. Bede, I. J. Rudas, and A. L. Bencsik, "First order linear fuzzy differential equations under generalized differentiability," Information Sciences, vol. 177, no. 7, pp. 1648-1662, 2007.

[16] M. L. Puri and D. A. Ralescu, "Differentials of fuzzy functions," Journal of Mathematical Analysis and Applications, vol. 91, no. 2, pp. 552-558, 1983.

[17] O. Kaleva, "Fuzzy differential equations," Fuzzy Sets and Systems, vol. 24, no. 3, pp. 301-317, 1987.

[18] H. Y. Goo and J. S. Park, "On the continuity of the Zadeh extensions," Journal of the Chungcheong Mathematical Society, vol. 20, no. 4, pp. 525-533, 2007.

[19] G. A. Anastassiou and S. G. Gal, "On a fuzzy trigonometric approximation theorem of Weierstrasstype," Journal of Fuzzy Mathematics, vol. 9, pp. 701-708, 2001.

[20] L. S. Chadli, A. Harir, and S. Melliani, "Fuzzy euler differential equation," SOP Transactions on Applied Mathematics, vol. 2, no. 1, 2015.

[21] J. Deng and Z. Deng, "Existence of solutions of initial value problems for nonlinear fractional differential equations," Applied Mathematics Letters, vol. 32, pp. 6-12, 2014.

[22] L. S. Chadli, A. Harir, and S. Melliani, "Solutions of fuzzy wave-like equations by variational iteration method," Annals of Fuzzy Mathematics and Informatics, vol. 8, no. 4, pp. 527-547, 2014.

[23] S. Melliani, A. Harir, and S. Chadli, "Solutions of fuzzy heatlike equations by variational iteration method," Annals of Fuzzy Mathematics and Informatics, vol. 10, no. 1, pp. 29-44, 2015. 